\title{
Non-contact Measurement of POA Irradiance and Cell Temperature for PV Systems
}

\author{
Li Hong Idris Lim \\ Department of Electronic Systems \\ University of Glasgow \\ Glasgow G12 8QQ, UK \\ Email: LiHonIdris.Lim@glasgow.ac.uk
}

\author{
Zhen Ye \\ REC Cells Pte Ltd. \\ 20 Tuas South Avenue 14 \\ Singapore 637312
}

\author{
Dazhi Yang \\ Singapore Institute of \\ Manufacturing Technology \\ 71 Nanyang Drive \\ Singapore 638075
}

\begin{abstract}
This paper presents a non-contact measurement of irradiance on plane of array (POA) and cell temperature for PV systems. The idea is motivated from the diode model of PV, where POA irradiance and cell temperature are proportional to the photocurrent and modified ideality factor, respectively. Based on the recent progress of diode model identification, the photocurrent and modified ideality factor can be linearly determined from $I-V$ characteristics, which makes it feasible to develop a non-contact measurement approach for POA irradiance and cell temperature, i.e., both of them will be derived completely from the diode mode parameter identification without the need of any sensors. The calibration of the proportional factors is done from the indoor module flash test and then applied to outdoor module testbed to show the accuracy and effectiveness of the proposed method.
\end{abstract}

\section{INTRODUCTION}

Irradiance on plane of array (POA) and cell temperature are important to PV systems because system performance, evaluated by performance ratio (PR), is derived from them. Usually, silicon sensors are applied in PV systems to measure the irradiance level on POA. Their structure is composed of a high-quality mono-crystalline solar cell connected to a high accuracy shunt, as shown in Fig.1, where $I_{L}$ is the photocurrent proportional to the POA irradiance, the diode represents the mono-crystalline cell, and $R_{s h}$ is the shunt. The low shunt $\left(R_{s h}=0.1 \Omega\right)$ causes the cell to operate close to the short-circuit point, which makes $I_{s h} \rightarrow I_{L}$ so that POA irradiance can be calibrated from $I_{s h}$ according to the proportionality.

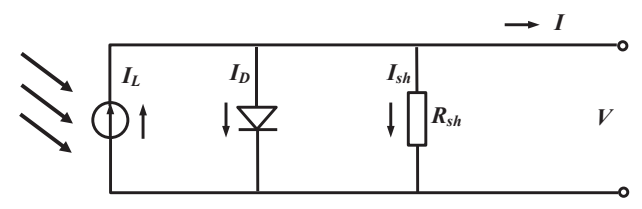

Fig. 1. Silicon sensor structure

Essentially, silicon sensors use an internal reference cell as a benchmark to sense the POA irradiance of PV modules/systems. The measurement accuracy highly depends on the differences between: 1) reference cell and PV modules; 2) $I_{L}$ and $I_{s h}$. However, mismatch between reference cell and PV modules is inevitable and $I_{L} \neq I_{s h}$ although compensation measures for temperature are taken into account. All of them cause the mismatch error up to $\pm 5 \%$, and the sensor needs to be recalibrated every two years to avoid the measurement shift caused by the degradation of reference cell.

A more accurate irradiance sensor is pyranometer, which covers the full spectrum of solar radiation $(300-2,800 \mathrm{~nm})$ from a field of view of 180 degrees. It is seldom deployed in PV systems due to: 1) much higher cost as compared to silicon sensor; 2) mismatch in spectrum as crystalline is not a full spectrum absorber; 3 ) is not applicable to measure POA irradiance.

Temperature measurement for PV systems is even worse than POA irradiance measurement because what is measured is not the true cell temperature but the temperature of the back sheet of modules. This is because cells are encapsulated between the layers of glass, EVA, back sheet during the process of lamination. However, it is also impractical to incorporate a sensor within the module, in direct contact with an individual cell, to measure the cell temperature. In addition, the nonuniformity of module temperature across the module area, which was assumed to be $\pm 1^{\circ} \mathrm{C}$ in [1], is not accounted for with this approach. The current compromise is to put a sensor attached to the back sheet, which causes the cell temperature measurement to be roughly $2-3^{\circ} \mathrm{C}$ lower than the true value. At a standard irradiance level of $1000 \mathrm{~W} / \mathrm{m}^{2}$, a mean cell-toback temperature difference of $2.5 \pm 1{ }^{\circ} \mathrm{C}$ was adopted in [2] for $\mathrm{c}-\mathrm{Si}$ modules with plastic back encapsulation.

It is much desired to find a more accurate way to measure the POA irradiance and cell temperature as more and more PV systems are installed all over the world, not only for the academic research, but also for the commercial investment evaluation. Motivated by the recent progress in the diode model parameter identification [3], [4], photocurrent $I_{L}$ and reverse saturation $I_{O}$ can be linearly determined from the $I-V$ characteristics of PV modules. Immediately, POA irradiance $G_{s}=\lambda I_{L}$, where $\lambda$ is a constant slope (to be calibrated) and independent of irradiance or temperature [5]. Cell temperature $T_{c}$ is derived from $I_{o}=B T_{c}^{3} \mathrm{e}^{-E_{g} /\left(k T_{c}\right)}$, where $E_{g}$ is the band gap of silicon and $B$ is a temperature independent constant [6]. No external sensors for irradiance or temperature is required once the $I-V$ curve is known.

The whole paper is organized as follows. Section II described the method to identify the parameters of PV diode model from the $I-V$ curve. Its application in non-contact measurement of POA irradiance and cell temperature is demonstrated in Section III and IV, for calibration and verification, 
respectively. Conclusions are drawn in Section V at last.

\section{Diode Model Parameter IdentificAtion}

A PV diode model is described by

$$
I=I_{L}-I_{o}\left(\mathrm{e}^{\frac{V+I R_{s}}{a}}-1\right)-\frac{V+I R_{s}}{R_{s h}}
$$

where $a$ is the modified ideality factor.

Let $y=I$ and $x=V+R_{s} I$, and (1) becomes

$$
y=I_{L}+I_{o}-I_{o} \mathrm{e}^{\frac{x}{a}}-\frac{x}{R_{s h}} .
$$

Taking differential once on both sides of (2) gives

$$
\frac{\mathrm{d} y}{\mathrm{~d} x}=-\frac{I_{o}}{a} \mathrm{e}^{\frac{x}{a}}-\frac{1}{R_{s h}} .
$$

Differentiating once more for (3) gives

$$
\frac{\mathrm{d}^{2} y}{\mathrm{~d} x^{2}}=-\frac{I_{o}}{a^{2}} \mathrm{e}^{\frac{x}{a}} .
$$

Eliminating $\mathrm{e}^{x / a}$ from (3) and (4) gives

$$
a \frac{\mathrm{d}^{2} y}{\mathrm{~d} x^{2}}-\frac{\mathrm{d} y}{\mathrm{~d} x}=\frac{1}{R_{s h}} .
$$

Let $t=x$ and $u(t) \equiv 1,(5)$ is equivalent to

$$
a \frac{\mathrm{d}^{2} y(t)}{\mathrm{d} t^{2}}-\frac{\mathrm{d} y(t)}{\mathrm{d} t}=\frac{u(t)}{R_{s h}},
$$

which is a standard differential equation representation of a second order linear system. $t$ is the "time", $u(t)$ and $y(t)$ are the system "input" and "output", respectively. Since $u(t) \equiv 1$, $y(t)$ is the unit step response of the system in "time" domain. Take Laplace transform, $F(s)=\mathcal{L}[f(t)]=\int_{0}^{\infty} \mathrm{e}^{-s t} f(t) \mathrm{d} t$, on both sides of (6),

$$
a\left[s^{2} Y(s)-s y(0)-y^{\prime}(0)\right]-[s Y(s)-y(0)]=\frac{U(s)}{R_{s h}} .
$$

Utilize $s U(s)=1$, and (7) is equivalent to

$$
\begin{aligned}
& a\left[s^{2} Y(s)-s^{2} U(s) y(0)-s U(s) y^{\prime}(0)\right] \\
& -[s Y(s)-s U(s) y(0)]=\frac{1}{R_{s h}} U(s) .
\end{aligned}
$$

It follows from (2) that $y(0)=I_{L}, y^{\prime}(0)=-I_{o} / a-1 / R_{s h}$, so the transfer function from $Y(s)$ to $U(s)$ is

$$
\begin{aligned}
G(s):=\frac{Y(s)}{U(s)} & =\frac{a y(0) s^{2}+\left[a y^{\prime}(0)-y(0)\right] s+\frac{1}{R_{s h}}}{a s^{2}-s} \\
& =\frac{a I_{L} s^{2}-\left(I_{o}+\frac{a}{R_{s h}}+I_{L}\right) s+\frac{1}{R_{s h}}}{a s^{2}-s} .
\end{aligned}
$$

The corresponding time domain differential equation is

$$
\begin{aligned}
a \frac{\mathrm{d}^{2} y(t)}{\mathrm{d} t^{2}}-\frac{\mathrm{d} y(t)}{\mathrm{d} t}= & a I_{L} \frac{\mathrm{d}^{2} u(t)}{\mathrm{d} t^{2}}-\left(I_{L}+I_{o}+\frac{a}{R_{s h}}\right) \\
& \times \frac{\mathrm{d} u(t)}{\mathrm{d} t}+\frac{u(t)}{R_{s h}} .
\end{aligned}
$$

It should be noted that (6) is different from (9) because of the non-zero initial conditions. In other words, (9) is the description of the same system of (6) but with zero initial conditions. This will facilitate the calculation of the integralbased identification shown as follows.

Define the double integral [7] as

$$
\int_{[0, t]}^{(2)} f(\tau)=\int_{0}^{t} \int_{0}^{\tau_{2}} f\left(\tau_{1}\right) \mathrm{d} \tau_{1} \mathrm{~d} \tau_{2} .
$$

Applying (10) to (9) gives

$$
\begin{aligned}
& a y(t)-a I_{L} u(t)+\left(I_{L}+I_{o}+\frac{a}{R_{s h}}\right) \int_{[0, t]}^{(1)} u(\tau) \\
& -\frac{1}{R_{s h}} \int_{[0, t]}^{(2)} u(\tau)=\int_{[0, t]}^{(1)} y(\tau) .
\end{aligned}
$$

Let $\gamma(t)=\int_{[0, t]}^{(1)} y(\tau), \theta=\left[a, a I_{L},\left(I_{L}+I_{o}+\frac{a}{R_{s h}}\right), \frac{1}{R_{s h}}\right]^{T}$, $\phi(t)=\left[y(t),-u(t), \int_{[0, t]}^{(1)} u(\tau),-\int_{[0, t]}^{(2)} u(\tau)\right]^{T}$, and (11) can be rewritten as the matrix format of $\phi^{T}(t) \theta=\gamma(t)$. Note that the matrix format holds for any $t_{i} \in[0, t], i=$ $1,2, \cdots, N$, where $N$ is the the number of data samples on the $I-V$ curve. This actually casts an equation group of $\Phi \theta=\Gamma$ with $\Phi=\left[\phi\left(t_{1}\right), \phi\left(t_{2}\right), \cdots, \phi\left(t_{N}\right)\right]^{T}$ and $\Gamma=\left[\gamma\left(t_{1}\right), \gamma\left(t_{2}\right), \cdots, \gamma\left(t_{N}\right)\right]^{T}$. Since $\Phi^{T} \Phi$ is proved to be nonsingular [3], the linear least square solution

$$
\theta=\left(\Phi^{T} \Phi\right)^{-1} \Phi^{T} \Gamma
$$

will minimise the square error of $(\Gamma-\Phi \theta)^{T}(\Gamma-\Phi \theta)$. Once $\theta$ is determined from (12), the parameters of one-diode model can be obtained by $a=\theta_{1}, I_{L}=\theta_{2} / \theta_{1}, I_{o}=\theta_{3}-\theta_{2} / \theta_{1}-\theta_{1} \theta_{4}$, $R_{s h}=1 / \theta_{4}$.

To calculate $\theta$ from (12), $\Phi$ and $\Gamma$ must be known. As both of them are integrals to $t, t$ must be known as well. Since $t=V+R_{s} I, R_{s}$ must be determined before applying integrals. It is clear to see that if $R_{s}$ is bigger than its real value, $t$ will increase so that the whole $I$ - $V$ curve will move to the right and the error between the real and estimated $I$ $V$ curves will be positive; If $R_{s}$ decreases, the whole $I-V$ curve will move to the left and the error between the real and estimated $I-V$ curves will be negative. Thus, $R_{s}$ can be used as a tuning parameter such that the root mean square error (RMSE) is minimised.

It is derived from (1) that

$$
-\frac{1}{\left.\frac{\mathrm{d} I}{\mathrm{~d} V}\right|_{o c}}=R_{s}+\frac{1}{\sum_{i=1}^{m} \frac{I_{o_{i}}}{a_{i}} \mathrm{e}^{\frac{V_{o c}}{a_{i}}}+\frac{1}{R_{s h}}}>R_{s},
$$

which implies the upper bound of $R_{s}$, i.e., $R_{s}^{u p p}=-1 /\left.\frac{\mathrm{d} I}{\mathrm{~d} V}\right|_{o c}$. The lower bound of $R_{s}$ can be zero at first, i.e., $R_{s}^{\text {low }}=0$. With such a band of $R_{s} \in\left[R_{s}^{\text {low }}, R_{s}^{\text {upp }}\right]$, binary search algorithm is applied to determine $R_{s}$ in the following way:

Step 1: Arbitrarily choose $R_{s}$ from $\left[R_{s}^{\text {low }}, R_{s}^{\text {upp }}\right]$ and calculate $\hat{a}_{i}, \hat{I}_{L}, \hat{I}_{o}$ and $\hat{R}_{s h}$ from the proposed linear least square (12);

Step 2: Calculate from (1) that

$$
\hat{y}(t)=\hat{I}_{L}-\hat{I}_{o}\left(\mathrm{e}^{\frac{V+I R_{s}}{\hat{\alpha}_{i}}}-1\right)-\frac{V+I R_{s}}{\hat{R}_{s h}},
$$

and $R M S E=\sqrt{\sum_{i=1}^{N}\left[\hat{y}\left(t_{i}\right)-y\left(t_{i}\right)\right]^{2} / N}$. 


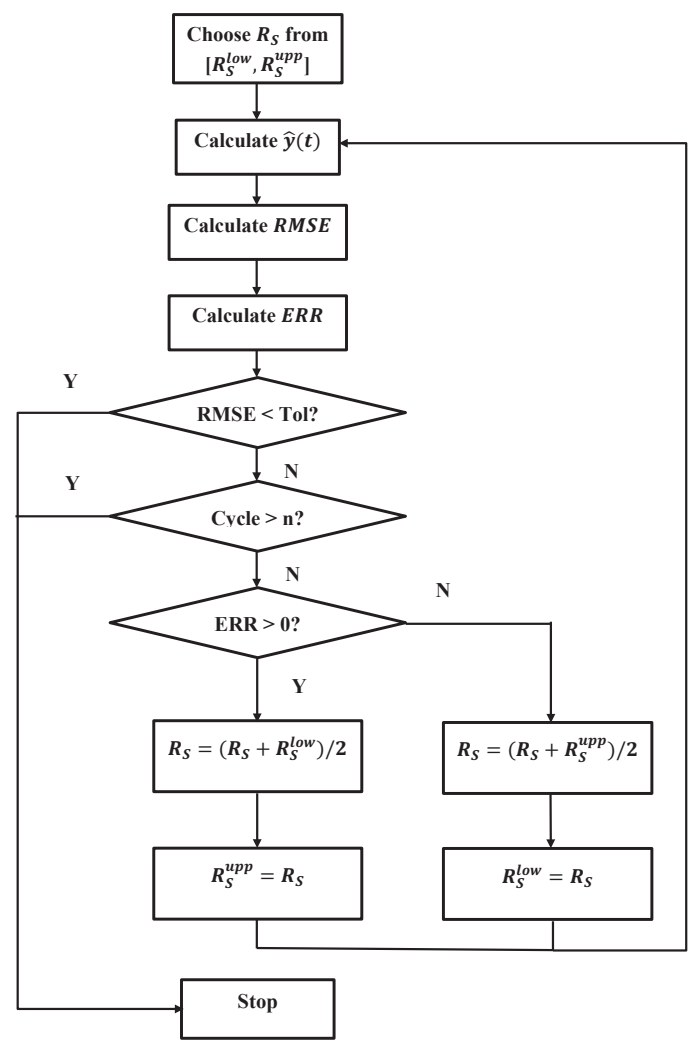

Fig. 2. Flowchart of the binary searching algorithm

Step 3: Calculate $E R R=\sum_{i=1}^{N}\left[\hat{y}\left(t_{i}\right)-y\left(t_{i}\right)\right]$. If $E R R>0$, adjust $R_{s}=\left(R_{s}+R_{s}^{\text {low }}\right) / 2$. Otherwise, adjust $R_{s}=\left(R_{s}+R_{s}^{u p p}\right) / 2$.

Step 4: Update $R_{s}^{\text {upp }}$ and $R_{s}^{\text {low }}$ according to the sign of $E R R$. If $E R R>0, R_{s}^{u p p}=R_{s}$, otherwise, $R_{s}^{\text {low }}=R_{s}$.

Step 5: If RMSE is less than some tolerance or the iterative cycle reaches some preset number, stop the searching. Otherwise, update $R_{s}^{u p p}$ and $R_{s}^{\text {low }}$ according to the sign of $E R R$ and go back to Step 2.

The flowchart of the binary searching is shown in Fig.2.

\section{INDOOR CALIBRATION}

In order to perform the calibration for both POA irradiance and cell temperature from the identified diode model, indoor flash tests were done on a PV module by the pulsed solar simulator (PASAN IIIB). This module was then mounted on an outdoor module testing bed for the validation of the proposed non-contact measurement. Note that the flash test time is very short (about $12 \mathrm{~ms}$ ) and the light intensity varies by less than $\pm 1 \%$. The intensity of the solar simulator is calibrated by Fraunhofer ISE with the overall uncertainty of module power measurement less than $\pm 2 \%$.

\section{A. Calibration of POA Irradiance}

As mentioned before, the photocurrent $I_{L}$ is proportional to POA irradiance $G_{s}$, i.e., $G_{s}=\lambda I_{L}$, and $\lambda$ is the slope. To calibrate $\lambda$, the $I-V$ characteristics of a full-sized commercial module were measured indoor by a PASAN IIIB with the constant illumination intensity of 200, 400, 600, 800, 1000, $1200 \mathrm{~W} / \mathrm{m}^{2}$. The temperature for such flash tests is fixed at $25^{\circ} \mathrm{C}$.

Fig. 3 shows the family $I-V$ characteristic of a PV module (crystalline) from the proposed indoor flash test, where estimation results by the identification method from Section II are indicated by circles. The estimation results obtained from the identified diode model parameters match closely to the $I-V$ curves from the indoor flash test. The identified diode model parameters and RMSE compared to the real $I-V$ curves are listed in Table I, which shows the accuracy of the proposed identification.

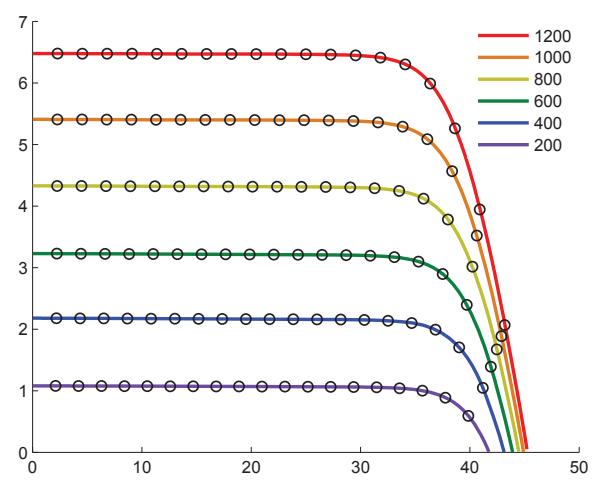

Fig. 3. Indoor flash test at different illumination intensity

TABLE I. IDENTIFICATION RESULTS

\begin{tabular}{c|ccccc|c}
\hline $\begin{array}{c}\text { Illumination } \\
\left(\mathrm{W} / \mathrm{m}^{2}\right)\end{array}$ & $\begin{array}{c}I_{L} \\
(\mathrm{~A})\end{array}$ & $\begin{array}{c}I_{o} \\
\left(10^{-9} \mathrm{~A}\right)\end{array}$ & $\begin{array}{c}a \\
(\mathrm{~V})\end{array}$ & $\begin{array}{c}R_{s} \\
(\Omega)\end{array}$ & $\begin{array}{c}R_{s h} \\
(\mathrm{k} \Omega)\end{array}$ & $\begin{array}{c}\text { RMSE } \\
\left(\times 10^{-3}\right)\end{array}$ \\
\hline 200 & 1.08 & 0.4782 & 1.9411 & 0.5293 & 1.8321 & 0.0849 \\
400 & 2.18 & 0.4757 & 1.9407 & 0.6278 & 1.3512 & 0.1410 \\
600 & 3.23 & 0.4745 & 1.9404 & 0.6339 & 1.3414 & 0.1809 \\
800 & 4.33 & 0.4741 & 1.9401 & 0.6345 & 1.5710 & 0.2130 \\
1000 & 5.41 & 0.4725 & 1.9399 & 0.6347 & 1.8408 & 0.2380 \\
1200 & 6.48 & 0.4786 & 1.9397 & 0.6347 & 2.1330 & 0.2569 \\
\hline
\end{tabular}

Based on the results from Table I, Fig 4 shows the correlation between $G_{s}$ and $I_{L}$. As expected, $I_{L}$ is proportional to $G_{s}$, which was discussed in I. The non-zero intercept is caused by measurement error, which brings the uncertainty of irradiance estimation up to $0.006 / 0.0054=1.11 \mathrm{~W} / \mathrm{m}^{2}$. The slope $\lambda$ from $G_{s}=\lambda I_{L}$ is determined by $\lambda=1 / 0.0054=185.1852$.

\section{B. Calibration of Cell Temperature}

Cell temperature is derived from $I_{o}=B T_{c}^{3} \mathrm{e}^{-E_{g} /\left(k T_{c}\right)}$, where $E_{g}$ is the band gap of silicon and $B$ is a temperature independent constant [6]. Both $B$ and $E_{g}$ are required to be calibrated. To do the calibration, the $I-V$ characteristics of the same module in Section III-A were measured by the PASAN IIIB in a thermal chamber. The illumination intensity is fixed at $1000 \mathrm{~W} / \mathrm{m}^{2}$ and the chamber temperature are set at $15^{\circ} \mathrm{C}$, $25^{\circ} \mathrm{C}, 35^{\circ} \mathrm{C}, 45^{\circ} \mathrm{C}, 55^{\circ} \mathrm{C}, 65^{\circ} \mathrm{C}$. 


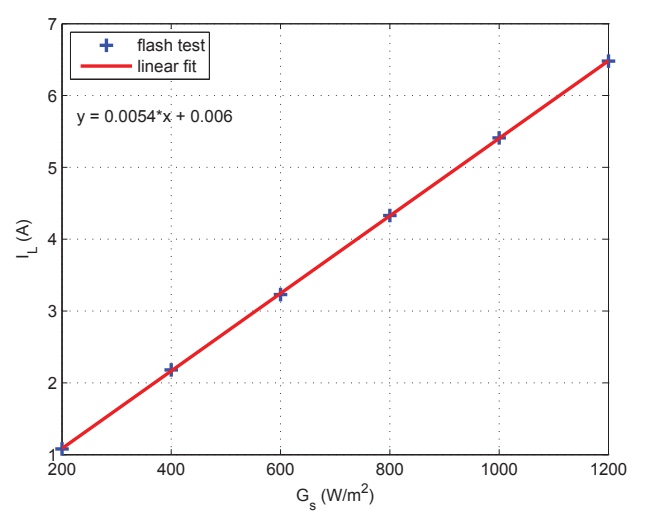

Fig. 4. Determination of $\lambda$ from $G_{s}=\lambda I_{L}$

Fig. 5 shows the results of the flash test at different temperature levels, where the circles represent the estimated $I$ $V$ curves by the proposed identification. The identified diode model parameters and RMSE compared to the real $I-V$ curves are listed in Table II.

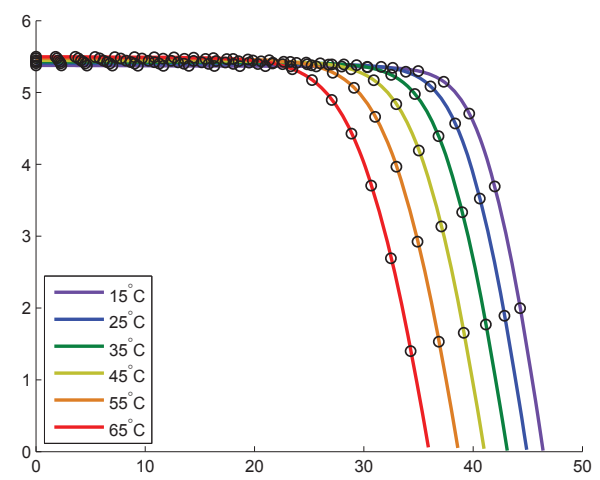

Fig. 5. Indoor flash test at different temperatures

TABLE II. IDENTIFICATION RESULTS

\begin{tabular}{c|ccccc|c}
\hline $\begin{array}{c}\text { Temperature } \\
\left({ }^{\circ} \mathrm{C}\right)\end{array}$ & $\begin{array}{c}I_{L} \\
(\mathrm{~A})\end{array}$ & $\begin{array}{c}I_{o} \\
\left(10^{-9} \mathrm{~A}\right)\end{array}$ & $\begin{array}{c}a \\
(\mathrm{~V})\end{array}$ & $\begin{array}{c}R_{s} \\
(\Omega)\end{array}$ & $\begin{array}{c}R_{s h} \\
(\mathrm{k} \Omega)\end{array}$ & $\begin{array}{c}\text { RMSE } \\
\left(\times 10^{-3}\right)\end{array}$ \\
\hline 15 & 5.38 & 0.0326 & 1.7970 & 0.6326 & 1.8486 & 0.2676 \\
25 & 5.41 & 0.4756 & 1.9399 & 0.6347 & 1.8409 & 0.2375 \\
35 & 5.43 & 5.8101 & 2.0883 & 0.6367 & 1.8335 & 0.2089 \\
45 & 5.45 & 61.544 & 2.2420 & 0.6378 & 1.8283 & 0.1810 \\
55 & 5.48 & 564.16 & 2.4012 & 0.6388 & 1.8180 & 0.1550 \\
65 & 5.50 & 4546.3 & 2.5659 & 0.6399 & 1.8075 & 0.1305 \\
\hline
\end{tabular}

With the identified $I_{o}$ from TableII, taking logarithmic to $I_{o}$ gives,

$$
\begin{aligned}
\ln I_{o} & =\ln B+3 \ln T_{c}-\frac{E_{g}}{k T_{c}}, \quad \Rightarrow \\
\ln I_{o}-3 \ln T_{c} & =-\frac{E_{g}}{k} T_{c}^{-1}+\ln B .
\end{aligned}
$$

Let $y=\ln I_{o}-3 \ln T_{c}, x=1 / T_{c}, \alpha=-E_{g} / k$ and $\beta=$ $\ln B$, (13) becomes $y=\alpha x+\beta$. The relationship between $x$ and $y$ are shown in Fig 6 . With linear fitting, $\alpha=-22122$ and $\beta=35.637$. Thus, $E_{g}=-k \alpha=3.0543 \times 10^{-19}$ and $B=\mathrm{e}^{\beta}=2.9988 \times 10^{15}$. After $E_{g}$ and $B$ are known, the cell temperature $T_{c}$ can be numerically determined by NewtonRaphson method with the initial $T_{c}=300 \mathrm{~K}$.

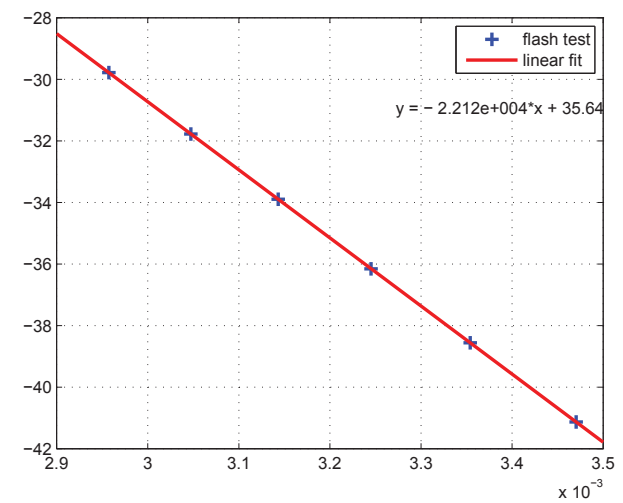

Fig. 6. Calibration of $E_{g}$ and $B$

\section{OUTDOOR VERIFICATION}

Outdoor module testing (OMT) is usually carried out by many PV panel manufacturers and solar research institutes for the module performance evaluation under the real operating environments. DC parameters including full $I-V$ curves, $V_{o c}$, $I_{s c}, V_{m p p}, I_{m p p}, P_{m p p}$ together with module temperature are measured and logged every minute. Environmental parameters including in-plane solar irradiance $G_{s}$, ambient temperature $T_{a m b}$, module temperature $T_{m o d}$, wind speed and wind direction are logged simultaneously with the DC parameters. Between $I-V$ measurements, electrical energy is maintained at the module maximum power point (MPP). The uncertainty of all electrical measured parameters is within $\pm 0.1 \%$ for full scale. With these $I-V$ data in time series, the diode model parameters can be identified online by the proposed method and correlated to the environmental factors like irradiance, temperature, etc.

To validate the proposed non-contact measurement for POA irradiance and cell temperature, the same module after the indoor calibration was put at OMT test bed for a whole day with the continuous recording of $I-V$ curves and meteorological data as shown in Fig.7.

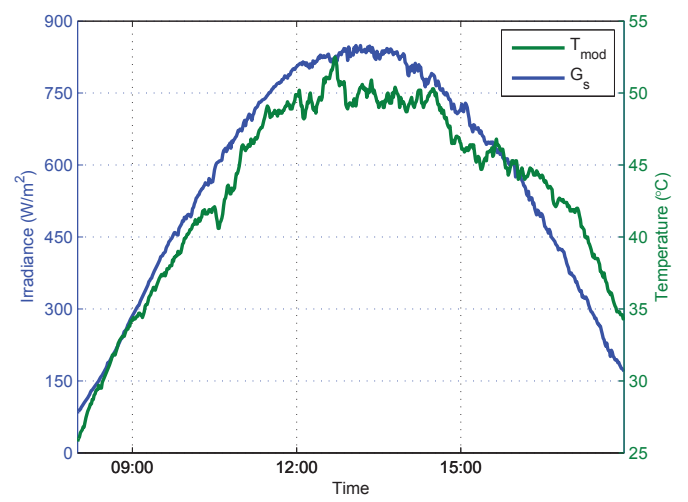

Fig. 7. Meteorological data of the same module after indoor calibration 
By applying the proposed method in Section II, the timevarying one-diode model parameters $I_{L}, I_{o}, a, R_{s}$ and $R_{s h}$ for the same day are identified, as shown in Fig.8. The variation of the identified parameters reflects the dynamics of the PV module under different environmental conditions, which cannot be seen from the static $I-V$ curves. With the identified diode model parameters, the POA irradiance and cell temperature can then be derived.
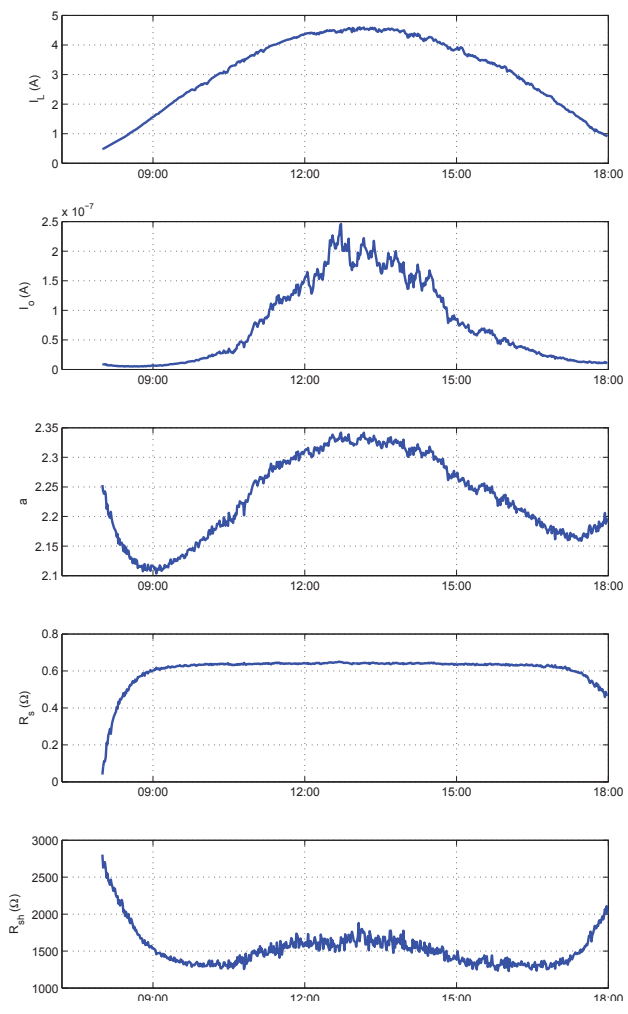

Fig. 8. Identified one-diode model parameters

\section{A. Comparison of POA Irradiance}

Based on the calibration value $\lambda$, the POA irradiance can be determined from $I_{L}$ by $G_{s}=\lambda I_{L}$. Fig.9 illustrates the comparison to the results from a reference silicon sensor which has the same inclined angle as the PV module. As seen from Fig 9, the non-contact measurement POA irradiance matches the irradiance measurement from the silicon sensor well.

\section{B. Comparison of Cell Temperature}

With the calibrated $E_{g}$ and $B$ from Section III-B, cell temperature $T_{c}$ is numerically determined by Newton-Raphson method. The comparison between $T_{c}$ and $T_{\text {mod }}$ (backsheet measurement) is shown in Fig.10. One can see that when irradiance increases in the morning, $T_{c}$ is usually higher than $T_{\text {mod }}$, which is due to the positive temperature gradient (from cell to backsheet) during that time. Whereas after solar

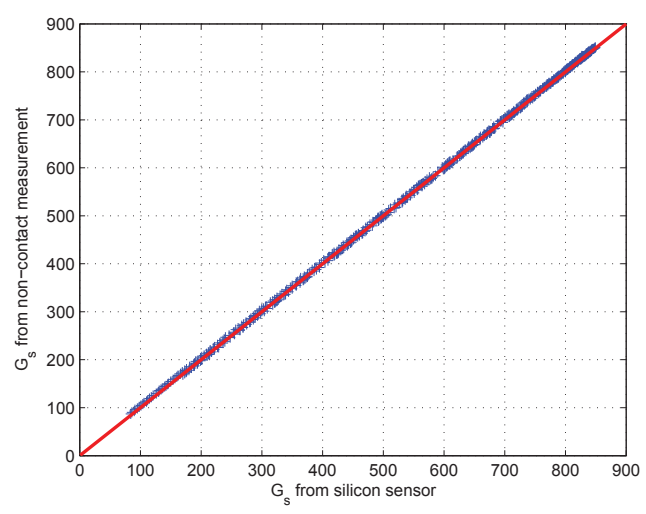

Fig. 9. POA irradiance: non-contact measurement vs. reference cell

noon when irradiance decreases, temperature gradient becomes negative due to the thermal delay, so $T_{c}$ is lower than $T_{\text {mod }}$. But the difference in between is within $\pm 2{ }^{\circ} \mathrm{C}$.

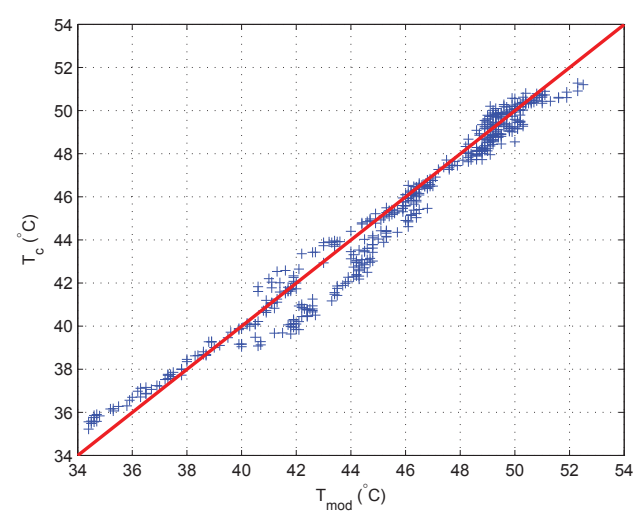

Fig. 10. Cell temperature: non-contact measurement vs. backsheet-attached sensors

\section{CONCLUSION}

A non-contact measurement for POA irradiance and cell temperature of PV panels is presented in this paper. The approach is based from the recent progress in diode model identification from I-V characteristics. The comparison to the conventional measurement of POA irradiance and module temperature shows the accuracy and reliability of the proposed non-contact measurement. It is suitable for OMT test and system monitoring to replace the existing meteorological sensors.

\section{REFERENCES}

[1] W. Z. H. Mullejans and R. Galleano, "Analysis and mitigation of measurement uncertainties in the traceability chain for the calibration of photovoltaic devices," Measurement Science and Technology, vol. 20, 2009.

[2] K. Whitfield and C. Osterwald, "Procedure for determining the uncertainty of photovoltaic module outdoor electrical performance," Progress in Photovoltaics: Research \& Applications, vol. 9, pp. 87 - 102, 2001.

[3] L. H. I. Lim, Z. Ye, J. Ye, D. Yang, and H. Du, "A linear identification of diode models from single $I-V$ characteristics of PV panels," IEEE Trans. Ind. Electron., 2015. 
[4] _ - "A linear method to extract diode model parameters of solar panels from a single $I$ - $V$ curve," Renewable Energy, vol. 76, pp. 135-142, 2015.

[5] W. De Soto, S. Klein, and W. Beckman, "Improvement and validation of a model for photovoltaic array performance," Sol. Energy, vol. 80, no. 1, pp. 78-88, 2006.

[6] G. H. Yordanov, O.-M. Midtgård, and T. O. Saetre, "Series resistance determination and further characterization of c-si PV modules," Renew. Energ., vol. 46, no. 10, pp. 72-80, 2012.

[7] Q.-G. Wang and Y. Zhang, "Robust identification of continuous systems with dead-time from step responses," Automatica, vol. 37, no. 3, pp. 377-390, 2001 